Effect of coherence and polarization on the polychromatic partially coherent dark hollow beam generated from axicon-lens system

This content has been downloaded from IOPscience. Please scroll down to see the full text.

2014 J. Opt. 16075402

(http://iopscience.iop.org/2040-8986/16/7/075402)

View the table of contents for this issue, or go to the journal homepage for more

Download details:

IP Address: 14.139.60.97

This content was downloaded on 24/09/2014 at 09:34

Please note that terms and conditions apply. 


\title{
Effect of coherence and polarization on the polychromatic partially coherent dark hollow beam generated from axicon-lens system
}

\author{
Stuti Joshi ${ }^{1,2}$, B K Yadav ${ }^{3}$, Mohd Shahid Khan ${ }^{2}$ and H C Kandpal ${ }^{1,3}$ \\ ${ }^{1}$ Quantum Optics \& Photon Physics, CSIR-National Physical Laboratory, Dr K. S. Krishnan Marg, \\ New Delhi-110012, India \\ ${ }^{2}$ Department of Physics, Jamia Millia Islamia, New Delhi-110025, India \\ ${ }^{3}$ Optical Radiation Standards, CSIR-National Physical Laboratory, Dr K. S. Krishnan Marg, \\ New Delhi-110012, India \\ E-mail: joshis@mail.nplindia.org
}

Received 2 March 2014, revised 21 April 2014

Accepted for publication 8 May 2014

Published 11 June 2014

\begin{abstract}
An experimental study is carried out to investigate the effect of coherence and polarization on the polychromatic partially coherent dark hollow beam (PCDHB). The experimental results show that the spatial coherence and source polarization affect the dark region of the generated hollow beam. The study shows that by varying the source degree of polarization (DOP), we get a tunable dark region. We find that the longer the spatial coherence length of the input beam, the larger the central dark size of the resultant PCDHB. Further, it is shown that polychromatic PCDHB with low spatial coherence travel a longer distance without being distorted than a beam with a high spatial coherence. These kinds of polychromatic beams may find potential application in the field of polychromatic light based free-space optical (FSO) communications.
\end{abstract}

Keywords: coherence, polarization, hollow beam, free-space optics

(Some figures may appear in colour only in the online journal)

\section{Introduction}

Dark hollow beams (DHBs) having zero on axis intensity in space surrounded by light have attracted much interest in the past decade owing to their potential applications in atomic optics [1], free-space optical (FSO) communication [2, 3] and biological sciences [4]. Various techniques have been proposed to generate hollow beams [5-7], and different kinds of hollow beams have been studied so far [3, 8-10]. It has been shown that the coherent light is sensitive to phase distortions and other optical aberrations $[11,12]$. In order to improve the quality of DHBs, partially coherent dark hollow beams (PCDHBs) have been introduced [6, 8]. These beams are less sensitive to speckles and less robust than coherent beams. In the last few years, a lot of attention has been paid to the study of PCDHBs because of their aforesaid advantages over completely coherent beams. Paraxial propagation properties of such beams have been studied in detail. It has been shown that the PCDHBs are less sensitive to phase distortion and are less affected by atmospheric turbulence [13, 14]. Thus, these beams might find potential applications in FSO communications.

Most of the previous experimental studies were devoted to monochromatic DHBs. Limited experimental work on polychromatic DHB have been reported so far. A few year ago, the first experimental study of polychromatic PCDHB was reported [2], and it was demonstrated that spectral anomalies in such types of beams might be exploited for FSO communications. Subsequently, the polychromatic PCDHB was generated by coupling a polychromatic beam emitted from a light-emitting diode into a multimode fibre [15].

In this paper, we report the results of our recent experimental work carried out to study coherence and source polarization effects on PCDHBs. The experimental results show that as we change the spatial coherence of the input beam, the size of the dark region of the resultant PCDHB 


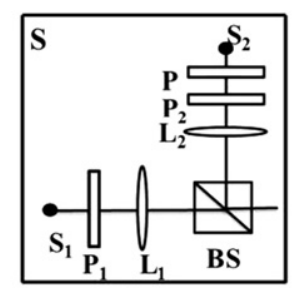

(a)

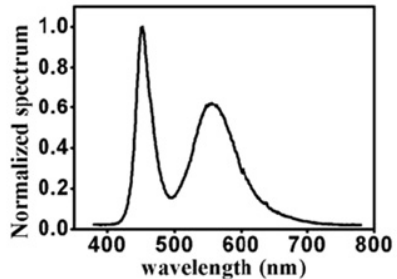

(c)

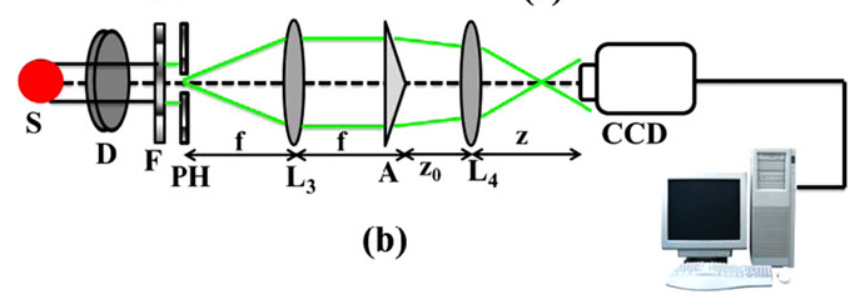

Figure 1. Schematic diagram of the experimental setup.

changes. Further, we get a tunable dark region for different values of the source polarization. Therefore, this study provides a flexible method for obtaining PCDHB with an adjustable and tunable dark region. Moreover, the observations show that the beam quality of the produced beam might be maintained by controlling the spatial coherence length of the partially coherent light.

\section{Experimental setup}

Figure 1 shows the experimental setup used to study the effect of source polarization and spatial coherence of the input beam on the polychromatic PCDHBs. The beams were produced using a well known axicon-lens system [7]. The inset (figure 1(a)) illustrates the arrangement to vary the degree of polarization (DOP) of the beam incident on an axicon. $S_{1}$ and $\mathrm{S}_{2}$ were white light LED sources (operated by a stabilized power supply) having almost the same spectral profiles. Light coming from $S_{1}$ and $S_{2}$ were made v-polarized and h-polarized by using polarizers $P_{1}$ and $P_{2}$, respectively. Lenses $L_{1}$ and $\mathrm{L}_{2}$ were used to collimate light coming from the polarizers $\mathrm{P}_{1}$ and $\mathrm{P}_{2}$. The collimated light further combines at the beam splitter (BS). This source arrangement part (figure 1(a)) was marked as $\mathrm{S}$ in figure 1(b). Light coming from the BS was passed through a diffuser (D) to get uniform intensity of the light incident on the pinhole. To get a Gaussian profile of the beam, a broadband filter $(\mathrm{F})$ with a peak wavelength at $560 \mathrm{~nm}$ was introduced before a pinhole $(\mathrm{PH})$. This way, a spatially incoherent, polychromatic source of uniform brightness and a Gaussian spectral profile was produced by illuminating a PH. The diameter of the $\mathrm{PH}$ was $0.5 \mathrm{~mm}$, and it was placed at the front focal plane of the lens $\mathrm{L}_{3}$. At the back focal plane of the lens $\mathrm{L}_{3}$ we got the partially coherent light [16] that impinged on an axicon A (made of BK7 glass). As a result, Bessel beams were generated [17] that were further focused by a positive lens $\mathrm{L}_{4}$ of focal length $f_{2}=120 \mathrm{~mm}$. This way, polychromatic PCDHBs were generated for $\mathrm{z}_{0}<f_{2}$, [7] where $z_{0}$ is the distance between axicon and lens $L_{4}$. In this experiment, the distance between axicon and lens $\mathrm{L}_{4}$ was

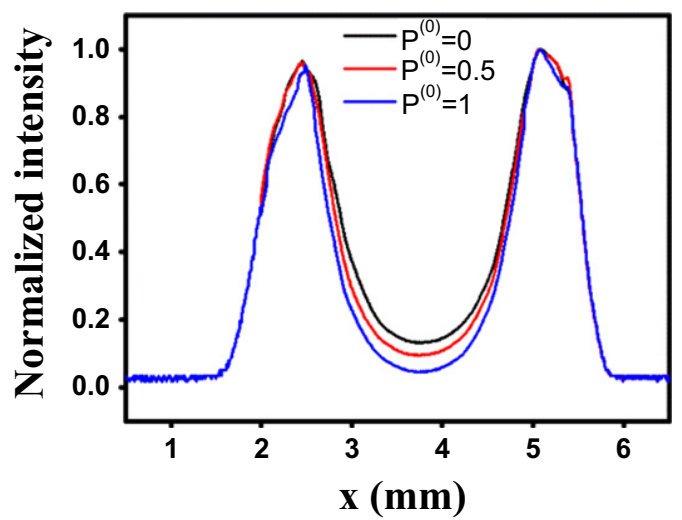

Figure 2. Variation in normalized intensity distributions of Polychromatic PCDHBs as function of $\mathrm{x}$ at $\mathrm{z}=150 \mathrm{~mm}$ for different DOP of the source.

adjusted $\left(\mathrm{z}_{0}=100 \mathrm{~mm}\right)$ to get a clear far field intensity distribution of the ring shaped beams. The transverse spatial distribution of the light intensity for different propagation distances ( $\mathrm{z}$ ) (the distance between lens $\mathrm{L}_{4}$ and CCD) was recorded by a computerized CCD camera (MicroPublisher 5.0 $\mathrm{RTV}, 3.4 \mu \mathrm{m} \times 3.4 \mu \mathrm{m}$ pixel size). Figure 2(c) is the spectrum of the white light (polychromatic) LED source.

\section{Results and discussion}

The DOP of the beam incident on the axicon lens was varied by putting a linear polarizer in front of the source $S_{2}$ (figure 1(a)) [18]. In these observations, the spatial coherence length of the beam incident on the axicon-lens was $L=0.0342 \mathrm{~mm}$. Figure 2 depicts the normalized intensity distributions of the polychromatic PCDHBs as a function of $\mathrm{x}$ (radial direction) at $\mathrm{z}=150 \mathrm{~mm}$ for different values of DOP, i.e., $P^{(0)}$. Figure 2 illustrates that maximum dip between two peaks of the intensity is obtained for completely polarized light, i.e., $P^{(0)} \approx 1$. The dip between two peaks of the intensity profile decreases as the DOP of the input beam decreases (or light becomes partially polarized). It is also observed that the width of the valley shrinks marginally as the DOP decreases, which confirms the theoretical prediction made in [19].

To study the coherence effects, the arrangement at the source was slightly changed. The experimental setup, as shown in figure 1(b), was used to study the effect of spatial coherence variation on the polychromatic PCDHB. For convenience; the DOP of the source was set at $P^{(0)} \approx 1$. It has been shown that to get $P^{(0)} \approx 1$, light coming from either source $\left(S_{1}\right.$ or $\left.S_{2}\right)$ should be zero [18]. Intensity of the beam coming from the polarizer $\mathrm{P}_{2}$ is nearly zero when the polarizer $\mathrm{P}$ and $\mathrm{P}_{2}$ are orthogonal. Now $\mathrm{S}$ was working as an incoherent light source. Light was made partially coherent at the back focal plane of the lens $\mathrm{L}_{3}$ by putting the source $S$ in the front focal plane of the lens $L_{3}$ [16]. The coherence length is estimated by the formula $L=\frac{3.832 \lambda f}{2 \pi d},[16]$ where $\lambda$ is the peak 

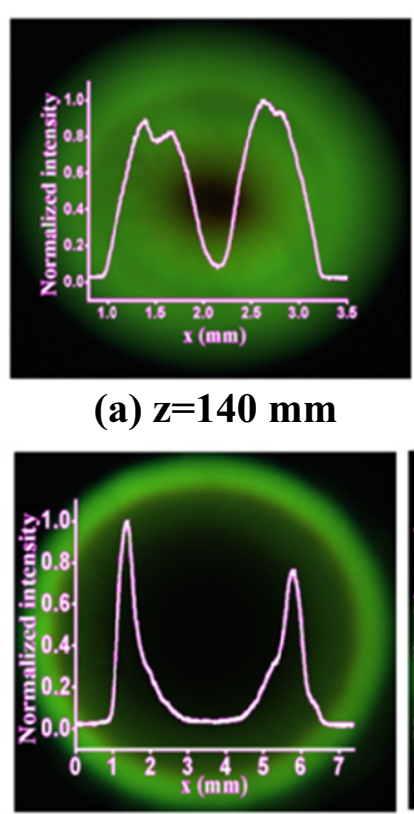

(c) $\mathrm{z}=170 \mathrm{~mm}$

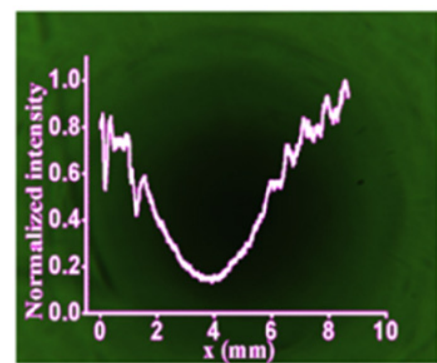

(e) $\mathrm{z}=230 \mathrm{~mm}$ (a) $\mathrm{z}=140 \mathrm{~mm}$

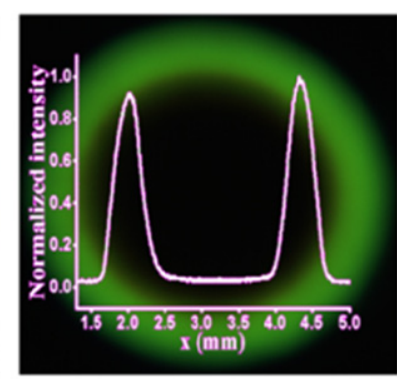

(b) $\mathrm{z}=150 \mathrm{~mm}$

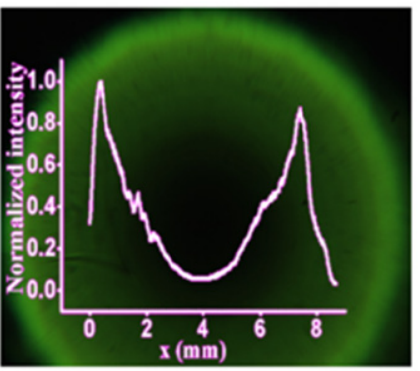

(d) $\mathrm{z}=190 \mathrm{~mm}$

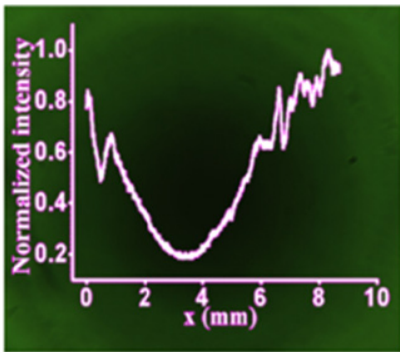

(f) $\mathrm{z}=\mathbf{2 5 0 \mathrm { mm }}$
Figure 3. Experimental results of the radial intensity distribution of polychromatic PCDHBs with propagation distance and the corresponding intensity profile for spatial coherence length $L=0.1025 \mathrm{~mm}$.

wavelength, $f$ is the focal length of lens $\mathrm{L}_{3}$ and $d$ is the diameter of the pinhole. (The FWHM of the source spectrum is $75 \mathrm{~nm}(525 \mathrm{~nm}-600 \mathrm{~nm})$. The value of $\mathrm{L}$ (for $f=50 \mathrm{~mm}$ ) in the extreme values vary from $0.0321 \mathrm{~mm}$ to $0.0366 \mathrm{~mm}$. These valuese are comparable to the value of $\mathrm{L}(0.0342 \mathrm{~mm})$ calculated for the peak wavelength $560 \mathrm{~nm}$. So the value of $\mathrm{L}$ at the peak wavelength vary $\pm 7 \%$ of the extreme values). This indicates that coherence length is directly proportional to the focal length. We used lenses $\left(\mathrm{L}_{3}\right)$ of different focal lengths to change the spatial coherence in order to achieve adjustable dark focus of the PCDHBs.

Figure 3 illustrates our experimental results of the transverse intensity distribution with corresponding intensity line profile of the polychromatic PCDHBs produced at different propagation distances $(\mathrm{z})$ for initial spatial coherence length $L=0.1025 \mathrm{~mm}$. Figure 4 shows the results for the case of $L=0.0342 \mathrm{~mm}$. The study indicates that the DHBs having a smooth beam profile are said to be high quality DHB [6]. When the distance between lens $\mathrm{L}_{4}$ and CCD is less than $170 \mathrm{~mm}$, we get a smooth beam profile of the PCDHBs for both the value of initial coherence lengths $L=0.1025 \mathrm{~mm}$ and (a) $\mathrm{z}=140 \mathrm{~mm}$

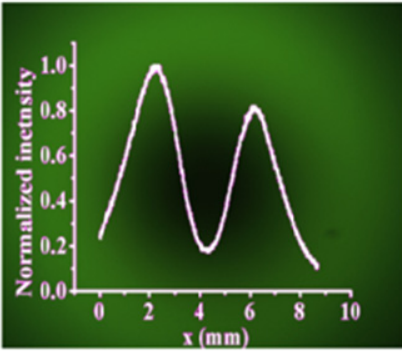

(c) $\mathrm{z}=170 \mathrm{~mm}$

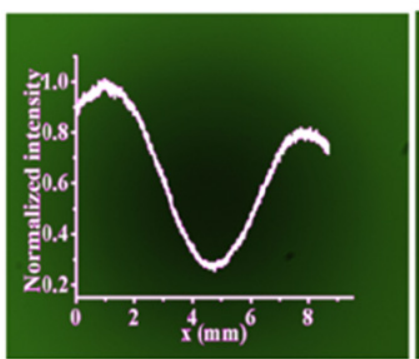

(e) $\mathrm{z}=230 \mathrm{~mm}$

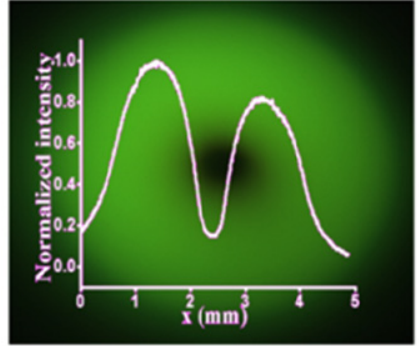

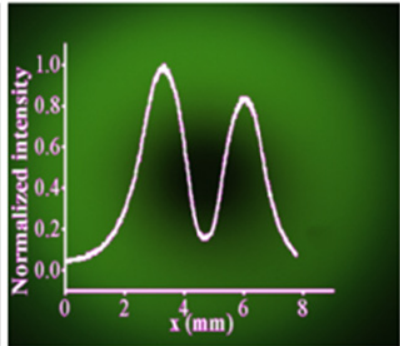

(b) $\mathrm{z}=150 \mathrm{~mm}$

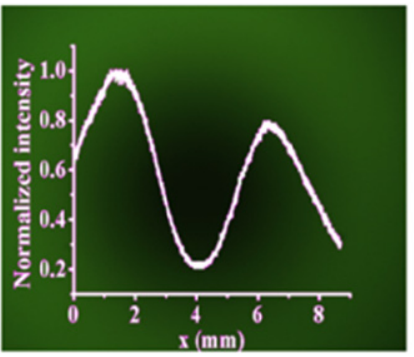

(d) $\mathrm{z}=190 \mathrm{~mm}$

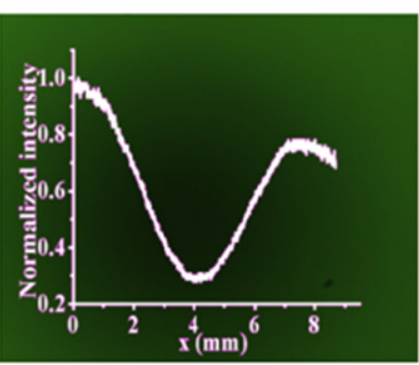

(f) $\mathrm{z}=\mathbf{2 5 0} \mathrm{mm}$
Figure 4. Experimental results of the radial intensity distribution of a polychromatic PCDHBs with propagation distance and the corresponding intensity profile for spatial coherence length $L=0.0342 \mathrm{~mm}$.

$L=0.0347 \mathrm{~mm}$, respectively. However, for $\mathrm{z}=175 \mathrm{~mm}$ or $\mathrm{z}>175 \mathrm{~mm}$, smooth beam profiles are obtained for only those PCDHBs for which the value of initial coherence is low. In other words, as the propagation distance $(\mathrm{z})$ increases, the beam with low spatial coherence might travel a longer distance without being distorted than the beams with high spatial coherence. In this way, the beam quality (or beam profile) would be maintained for a longer propagation distance. Smooth beam profile is the essence of low coherence as speckles in the generated hollow beam also decrease. Our results agree with that reported earlier $[6,8]$.

The effect of spatial coherence on the dark region of the generated polychromatic PCDHB for $P^{(0)} \approx 1$ is shown in figure 5. Figure 5(a) represents the intensity distribution of the PCDHB in the $x-y$ plane for different spatial coherence length at $\mathrm{z}=150 \mathrm{~mm}$. Figure 5(b) depicts the corresponding normalized intensity line profiles. One can notice from figure 5 that the size of the dark region increases as the spatial coherence length of the input beam increases. The size of the dark region for coherence lengths $0.0683 \mathrm{~mm}, 0.1025 \mathrm{~mm}$, $0.1367 \mathrm{~mm}$ and $0.1708 \mathrm{~mm}$, respectively, were calculated to 


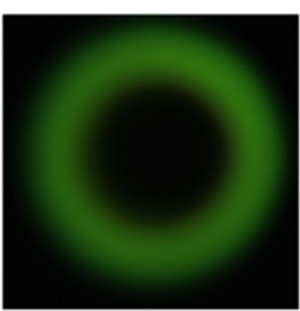

$\mathrm{L}=\mathbf{0 . 0 6 8 3} \mathrm{mm}$

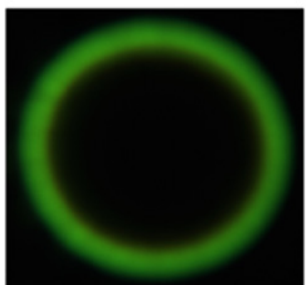

$\mathrm{L}=\mathbf{0 . 1 2 3 6 7} \mathrm{mm}$

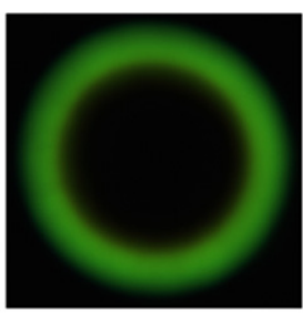

$\mathrm{L}=\mathbf{0 . 1 0 2 5} \mathbf{~ m m}$

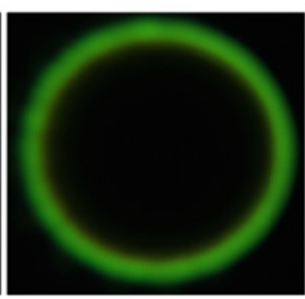

$\mathrm{L}=\mathbf{0 . 1 7 0 9} \mathbf{m m}$

(a)

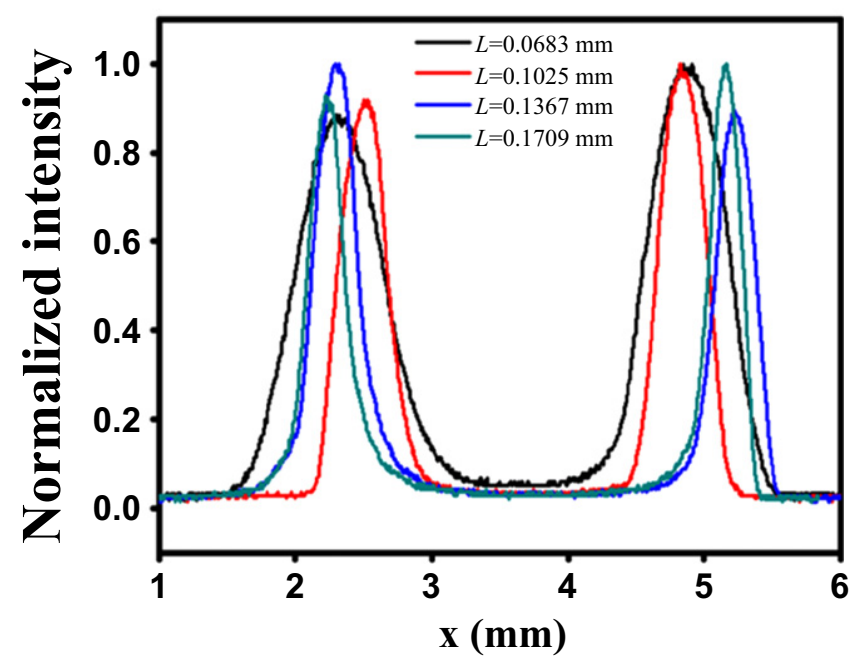

(b)

Figure 5. Intensity distribution of polychromatic PCDHBs at $\mathrm{z}=150 \mathrm{~mm}$ for different spatial coherence length (b) Curves for normalized intensity distributions as a function of transverse distance $\mathrm{x}$.

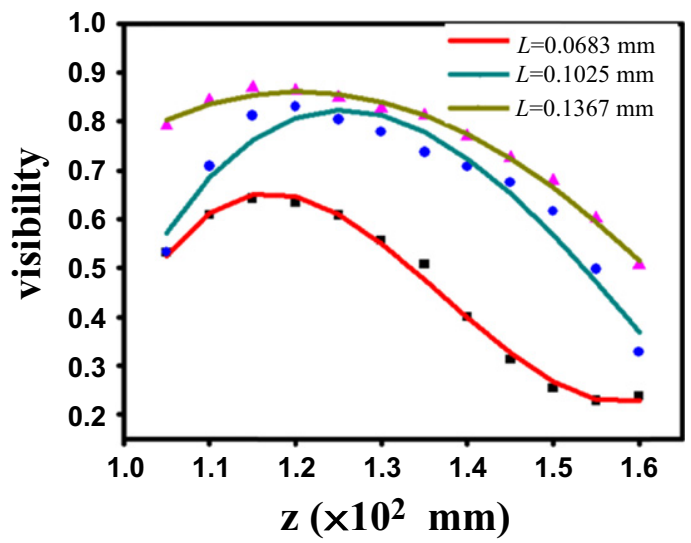

Figure 6. Experimentally measured visibility of the Polychromatic PCDHBs as a function of propagation distance (z) for different spatial coherence length.

decay in the visibility was due to the occurrence of speckles in the beam with high spatial coherence length.

\section{Conclusion}

In this experimental investigation, we observed that the beams with low spatial coherence travel a longer distance in freespace without being distorted. It is also noticed that the dark region of the polychromatic PCDHBs depends on the spatial coherence of the incident beam as well as on the source polarization. We illustrated these properties by showing intensity distributions and corresponding intensity profiles, which indicate the change in the dark region. We have shown that the height of the valley between two intensity peaks and the width of the valley increases as the DOP increases. Further, it is shown that the width of the dark region also get influenced by the spatial coherence. These kinds of hollow beams are less sensitive to speckles and show advantages over a completely spatially coherent hollow beam. Therefore, such types of beams might find potential applications in short range and indoor FSO communications.

\section{Acknowledgement}

S Joshi acknowledges the Council of Scientific and Industrial Research, New Delhi, India for financial support as Senior Research Fellowship. The authors thank the Director, CSIRNational Physical Laboratory for giving permission to publish the paper.

\section{References}

[1] Yin J, Zhu Y, Jhe W and Wang Z 1998 Atom guiding and cooling in a dark hollow laser beam Phys. Rev. A 58509

[2] Yadav B K and Kandpal H C 2011 Spectral anomalies of polychromatic DHGB and its applications in FSO J. Lightwave Technol. 29 960-6 
[3] Cai Y and He S 2006 Propagation of various dark hollow beams in a turbulent atmosphere Optics Express 14 1353-67

[4] Grier D G 2003 A revolution in optical manipulation Nature 424 810-6

[5] Fatemi F K and Bashkansky M 2006 Generation of hollow beams by using a binary spatial light modulator Opt. Lett. 31 864-6

[6] Zhao C, Cai Y, Wang F, Lu X and Wang Y 2008 Generation of a high-quality partially coherent dark hollow beam with a multimode fiber Opt. Lett. 33 1389-91

[7] Wei M-D, Shiao W-L and Lin Y-T 2005 Adjustable generation of bottle and hollow beams using an axicon Opt. Commun. 248 7-14

[8] Lü X and Cai Y 2007 Partially coherent circular and elliptical dark hollow beams and their paraxial propagations Phys. Lett. A 369 157-66

[9] Li X and Cai Y 2010 Nonparaxial propagation of a partially coherent dark hollow beam Appl. Phys. B 102 205-13

[10] Pu J, Dong M and Wang T 2006 Generation of adjustable partially coherent bottle beams by use of an axicon-lens system Appl. Opt. 45 7553-6

[11] Roichman Y, Waldron A, Gardel E and Grier D G 2006 Optical traps with geometric aberrations Appl. Opt. 45 3425-9

[12] Singh R K, Senthilkumaran P and Singh K 2007 The effect of astigmatism on the diffraction of a vortex carrying beam with a Gaussian background J. Opt. A: Pure Appl. Opt. 9543

[13] Eyyuboğlu H T 2008 Propagation and coherence properties of higher order partially coherent dark hollow beams in turbulence Opt. Laser Technol. 40 156-66

[14] Yuan Y, Cai Y, Qu J, Eyyuboglu H T, Baykal Y and Korotkova O 2009 M2-factor of coherent and partially coherent dark hollow beams propagating in turbulent atmosphere Opt. Express 17 17344-56

[15] Zhu S, Zhao C, Chen Y and Cai Y 2013 Experimental generation of a polychromatic partially coherent dark hollow beam Optik.-IJLEO 124 5271-3

[16] Foley J T 1991 Effect of an aperture on the spectrum of partially coherent light JOSA A 8 1099-105

[17] Herman R and Wiggins T 1991 Production and uses of diffractionless beams JOSA A 8 932-42

[18] Joshi S, Yadav B, Verma M, Khan M S and Kandpal H 2013 Effect of polarization on spectral anomalies of diffracted stochastic electromagnetic beams J. Opt. 15035405

[19] Long X, Lu K, Zhang Y, Guo J and Li K 2011 Effect of polarization on the evolution of electromagnetic hollow Gaussian Schell-model beam Opt. Commun. 284 715-8

[20] Shvedov V G, Izdebskaya Y V, Rode A V, Desyatnikov A S, Krolikowski W and Kivshar Y S 2008 Generation of optical bottle beams by incoherent white-light vortices Opt. Express 16 20902-7 